\title{
Role of Ultrasonography in Diagnosis and Treatment of Frozen Shoulder
}

\author{
Gi-Young Park \\ Department of Rehabilitation Medicine, Daegu Catholic University School of Medicine, Daegu, Korea
}

Frozen shoulder (FS) is a common, painful and disabling condition of the shoulder. Patients usually present with an insidious onset of symptoms with gradual restriction and loss of shoulder mobility. FS can be categorized into primary and secondary types. The natural course of FS is characterized by the following 3 stages: the painful, the freezing/frozen, and the thawing phase based on the duration of symptoms, as well as pain and limitation of motion observed on physical examination. Diagnosis of FS is based on careful and accurate history taking and physical examination. Imaging modalities including arthrography, ultrasonography, and magnetic resonance imaging are useful in excluding concomitant painful conditions of the shoulder and in confirming FS. Ultrasonography is recommended as the first-line imaging modality to diagnose FS because it is noninvasive, it provides an easy comparison of ultrasonography parameters between the affected and unaffected sides, and it reflects the clinical characteristics of FS. The goal of treatment in patients with FS is pain reduction and restoration of normal function and mobility of the shoulder. Ultrasonography-guided glenohumeral joint injection, suprascapular nerve block, and distention arthrography achieve favorable therapeutic outcomes by virtue of greater accuracy. Ultrasonography and ultrasonography guided injections can accurately diagnose and effectively treat patients with FS. (J Rheum Dis 2019;26:149-155)

Key Words. Frozen shoulder, Ultrasonography, Diagnosis, Treatment

\section{INTRODUCTION}

Frozen shoulder (FS), also referred to adhesive capsulitis, is characterized by the gradual onset of pain with limitation of shoulder motion. The disabling combination of shoulder pain with restricted movement was first described by Duplay [1] in 1872. He used the term periarthritis to describe painful stiffening of the shoulder, which was attributed to inflammation of the subacromial bursa. The term FS was subsequently introduced by Codman [2] to describe the typical symptoms of FS including an insidious onset of shoulder pain, pain around the insertion of the deltoid muscle, inability to lie on the affected side, and painful limitation of both active and passive external rotation and elevation despite normal radiographs of the shoulder. Codman [2] further stated that although the etiology of FS remained uncertain, the disorder was difficult to define, treat, and explain from a pathology perspective. However, it was a condition that would certainly resolve over time. Neviaser [3] was the first to describe the pathology of the disorder including inflammation, fibrosis, contracture of the shoulder capsule, and adherent axillary fold of the humeral head, thus causing limited joint mobility, and suggested the term adhesive capsulitis.

\section{MAIN SUBJECTS}

\section{Classification}

The FS is classified into primary and secondary types [4]. Primary FS shows idiopathic onset and is defined as an idiopathic global limitation of humeroscapular motion

Received : March 23, 2019, Revised : April 16, 2019, Accepted : April 17, 2019

Corresponding to : Gi-Young Park (iD http://orcid.org/0000-0003-0056-1556

Department of Rehabilitation Medicine, Daegu Catholic University School of Medicine, 33 Duryugongwon-ro 17-gil, Nam-gu, Daegu 42472, Korea. E-mail : parkgy@cu.ac.kr 
resulting from contracture and loss of compliance of the glenohumeral joint capsule. Secondary FS is associated with known pathology and is further classified into intrinsic, extrinsic, and systemic subtypes [5]. Conditions such as rotator cuff tendinosis and tears, and calcific tendinitis precede intrinsic secondary FS. Secondary extrinsic FS may be associated with post-trauma, iatrogenic (postsurgical), high impact force or low-level repetitive activity. Systemic secondary FS occurs in patients with co-morbidities such as diabetes, thyroid abnormalities or cardiac disease.

\section{Pathology}

The pathological changes associated with FS include chronic synovial inflammation followed by fibroticcontracture of the rotator interval, the glenohumeral capsule, and ligaments. Therefore, FS can be considered is a soft tissue affliction, not a bone disorder, which limits glenohumeral joint motion secondary to shortening and thickening of soft tissues [3].

\section{Stages}

The natural course of FS characteristically shows the following 3 stages of evolution: the painful, stiff, and resolution phase [6]. Hannafin and Chiaia [7] described 4 stages including a preadhesive, freezing, frozen, and thawing phase based on duration of symptoms, pain and limitation of motion, physical examination under anesthesia, and arthroscopic findings. The mean duration of symptoms in patients with FS is 30.1 months (range 12 42 months). Some degree of motion limitation, particularly in internal rotation, persists in patients during the thawing phase; however, minimal functional limitations are observed. Determination of these stages is essential to administer appropriate treatment, predict the prog- nosis, and establish appropriate patient and physician expectations. The staging of FS is determined clinically based on symptoms and physical examination. Intra-articular injection of local anesthetic is useful to distinguish between the painful and the stiff stages. Early diagnosis of FS shortens the duration of shoulder stiffness, thereby significantly reducing the patient's morbidity.

\section{Diagnosis}

FS can usually be diagnosed on the basis of accurate history taking and clinical findings. However, FS may mimic other causes of shoulder pain including arthritis, rotator cuff disorders, calcific tendinitis, and impingement syndrome. Cyriax [8] described atypical pattern of progressively restricted shoulder joint movement attributed to capsular restriction that was termed a capsular pattern of range-of-motion restriction. The capsular pattern of limitation is described as maximal limitation of external rotation, followed by limitation of abduction, which is more significantly limited than internal rotation.

Normal radiographs are needed to exclude other painful shoulder disorders that may also present with painful restriction of shoulder motion. Therefore, in addition to radiographs, imaging modalities such as arthrography, ultrasonography, and magnetic resonance imaging (MRI) are required to exclude other painful concurrent disorders and to confirm FS. Previously, conventional arthrography was used as a standard diagnostic modality for FS. It reveals a small volume capacity of the glenohumeral joint $(7 \sim 10 \mathrm{~mL})$, synovial irregularities and serrations of the joint capsule, non-filling of long head of the bicipital tendon sheath, and obliteration of the subcapsular or axillary recess (AR) $[9,10]$.

Recently, the following characteristic MRI findings are used to successfully diagnose FS: complete obliteration of
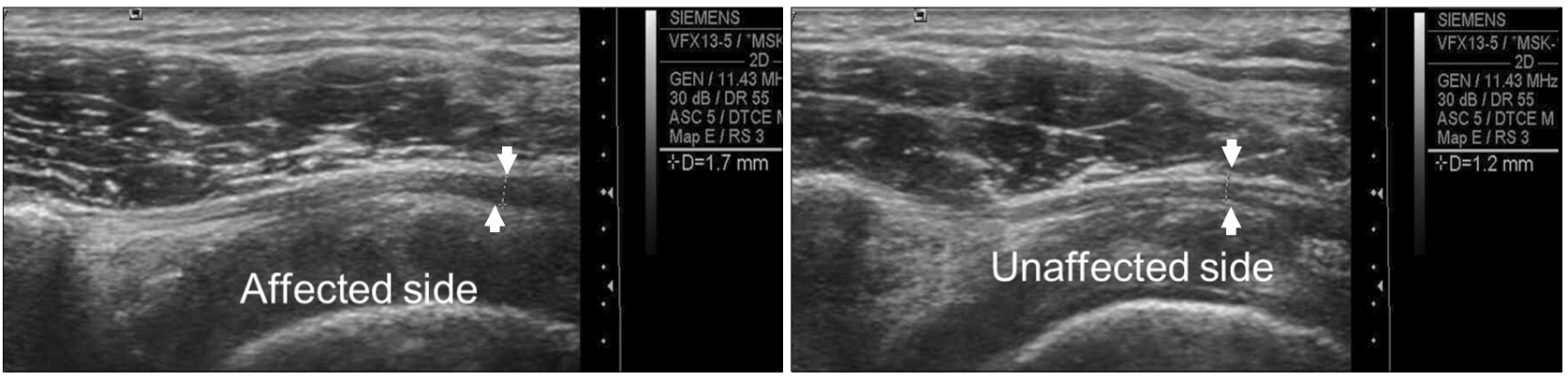

Figure 1. Ultrasonography measurement of coracohumeral ligament thickness. Longitudinal ultrasonography scan shows that the coracohumeral ligament thickness of the affected shoulder is thicker than that of the unaffected shoulder in the patient with frozen stage frozen shoulder. 
the fat triangle under the coracoid process, thickened coracohumeral ligament, increased signal intensity of the joint capsule and synovial membrane in the rotator cuff interval and AR with gadolium-chelate enhancement using indirect (intravenous) MR arthrography $[11,12]$.

Ultrasonography can accurately identify soft tissue changes such as coracohumeral ligament and AR thickening, and the presence of fibro-inflammatory soft tissue in the rotator cuff interval in patients with FS. Ultrasonography measurement of coracohumeral ligament thickness is useful to diagnose FS $[13,14]$ (Figure 1). Previous studies reported that power Doppler ultrasonography showed hypoechogenicity surrounding the long head of the biceps tendon with increased vascularity at the rotator cuff interval in all patients with FS $[15,16]$ (Figure 2). However, another study reported that power Doppler ultrasonography showed increased vascularity in the rotator cuff interval in only $29 \%$ of patients with FS

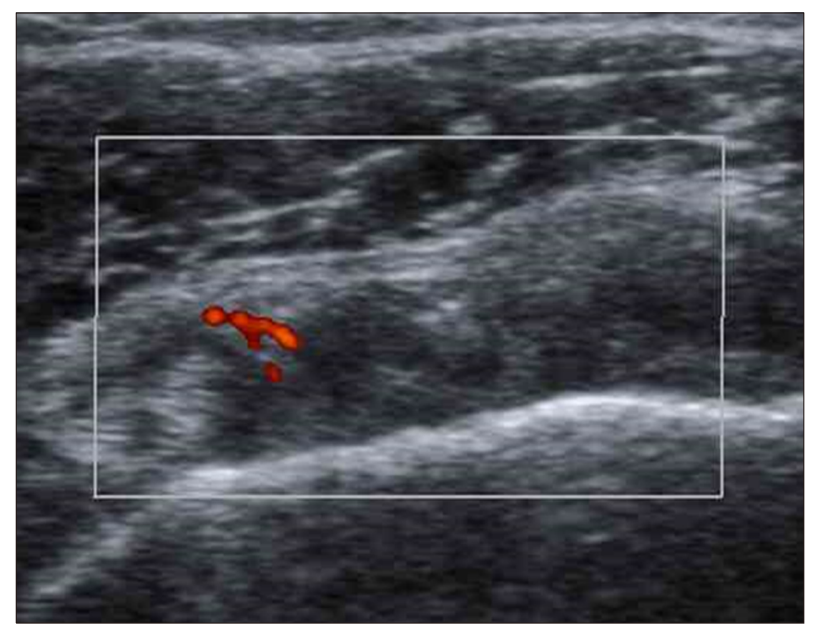

Figure 2. Transverse power Doppler ultrasonography scan shows hypervascularity in rotator cuff interval.
[17]. In a recent study increased vascularity was observed in the rotator cuff interval in only 10 of 75 patients (13.3\%) during the freezing or frozen stage of FS. Therefore, the validity of Doppler ultrasonography in the diagnosis of FS remains controversial [18]. Sonoelastography is a recently developed technique based on real-time ultrasonography for evaluation of tissue elasticity. This technique is based on the principle that pressure on tissues produces changes in those tissues depending upon their elasticity and that tissue compression produces a certain degree of strain (displacement), which is lesser in hard tissues and greater in softer tissues [19]. Pervious report investigating patients with FS have described that shear wave sonoelastography revealed increased stiffness of the thickened coracohumeral ligament in the affected shoulder compared with that in the unaffected shoulder [20]. This finding suggests that increased stiffness of the coracohumeral ligament may contribute to the limited range of shoulder external rotation. Ultrasonography measurements of the AR thickness in asymptomatic shoulders showed high intra- and inter-rater reliability; thus ultrasonography is a useful imaging modality to measure the AR thickness (Figure 3). Moreover, the AR thickness was shown to vary with sex, height, and weight of subjects [21]. Therefore, measurement of the AR thickness in the affected shoulder is not recommended as a good diagnostic indicator to diagnose FS. The ratio of the AR thickness between the affected and the unaffected shoulders could serve as a reliable diagnostic standard in the patients with unilateral FS. Comparison of imaging findings using arthrography, ultrasonography, and MRI with clinical impairment in patients with the freezing or frozen stage of FS showed that ultrasonography and arthrographic findings were correlated with clinical assessment scores. However, not all
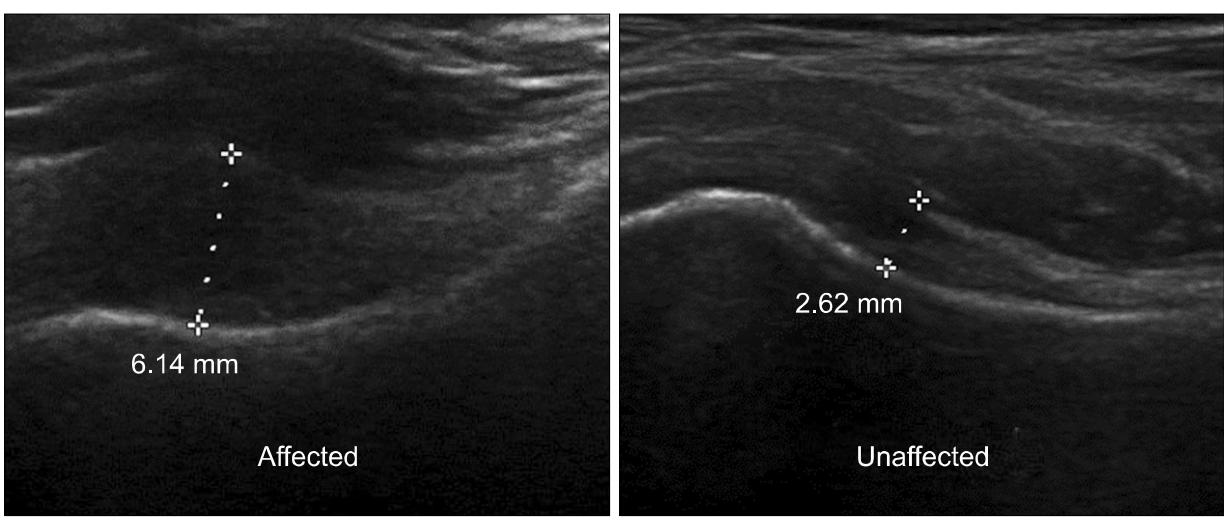

Figure 3. Ultrasonography measurement of axillary recess thickness. Longitudinal ultrasonography scan shows that the axillary recess thickness of the affected shoulder is thicker than that of the unaffected shoulder in the patient with frozen stage frozen shoulder. Axillary recess ratio is $2.34(6.14 / 2.62 \mathrm{~mm})$. 
MRI parameters including the thickness of the coracohumeral ligament, AR, and enhanced portion of the rotator cuff interval showed such a correlation [18]. Although arthrography and ultrasonography are complementary, ultrasonography measurements of AR and coracohumeral ligament thickness are recommended to diagnose FS for the following reasons; ultrasonography is a noninvasive modality, it is easy to compare ultrasonography parameters between the affected and the unaffected sides, it reflects the clinical characteristics of FS, and ultrasonography parameters serve as reliable anatomical indicators of FS.

\section{Treatment}

The goal of treatment in patients with FS is pain reduction and restoration of normal function and motion of the shoulder. Conservative treatments for FS include patient education, oral administration of nonsteroidal anti-inflammatory drugs and opioids, physical therapy, therapeutic exercise, joint mobilization and manipulation, intra-articular corticosteroid and hyaluronic acid injections, suprascapular nerve block, glenohumeral distention arthrography, manipulation under general anesthesia, interventional microadhesiolysis, and translational mobilization with serial capsular distention. Decision-making regarding treatment of FS warrants careful attention to the patient's symptoms and the stage of the disease because patients with FS require an individualized therapeutic strategy according to patient's needs. Prior to treatment initiation, patients should be categorized into the 'more pain than limitation of shoulder mobility' or 'more limitation than pain' groups.

\section{1) Ultrasonography-guided Intra-articular corticosteroid injection}

Intra-articular corticosteroid injection and suprascapular nerve block are effective in patients with more pain than limitation of shoulder mobility. Intra-articular corticosteroid injection to reduce inflammation, relieve pain, and improve the efficacy of a stretching exercise program [22]. Blind intra-articular injection (without imaging guidance) may be inaccurate in many cases, and accurate injection is required to accomplish favorable clinical outcomes. Therefore, ultrasonography-guided glenohumeral joint injection is useful in this context. Ultrasonography-guided glenohumeral joint injection can be performed with the patient in a seated or prone position, using the anterior, posterior, and rotator cuff interval approaches. The author personally prefers the posterior approach with the patient in a prone position because it is easier for the ultrasonographer, particularly a beginner to fix the transducer during injection in the prone position than in the seated position (Figure 4A and 4B). The patient is placed in the prone position with the shoulder in a position of internal rotation with a pillow placed beneath the shoulder. The needle tip is advanced to target the point between the posterior labrum and the posterior humeral head cartilage (Figure 5). The previous study have reported that a glenohumeral corticosteroid injection did not differ from a subacromial subdeltoid bursa corticosteroid injection with respect to therapeutic effectiveness and that a subacromial subdeltoid injection combined with a glenohumeral injection is a useful alternative to treat FS [23].

\section{2) Ultrasonography-guided Suprascapular nerve block} The suprascapular nerve is a mixed nerve that originates from the upper trunk of the brachial plexus, which is formed by the union of the ventral rami of the $\mathrm{C} 5$ and $\mathrm{C} 6$ nerve roots. It provides motor innervation to the supraspinatus and infraspinatus muscles of the rotator cuff and sensory innervation to approximately $70 \%$ of the gleno-
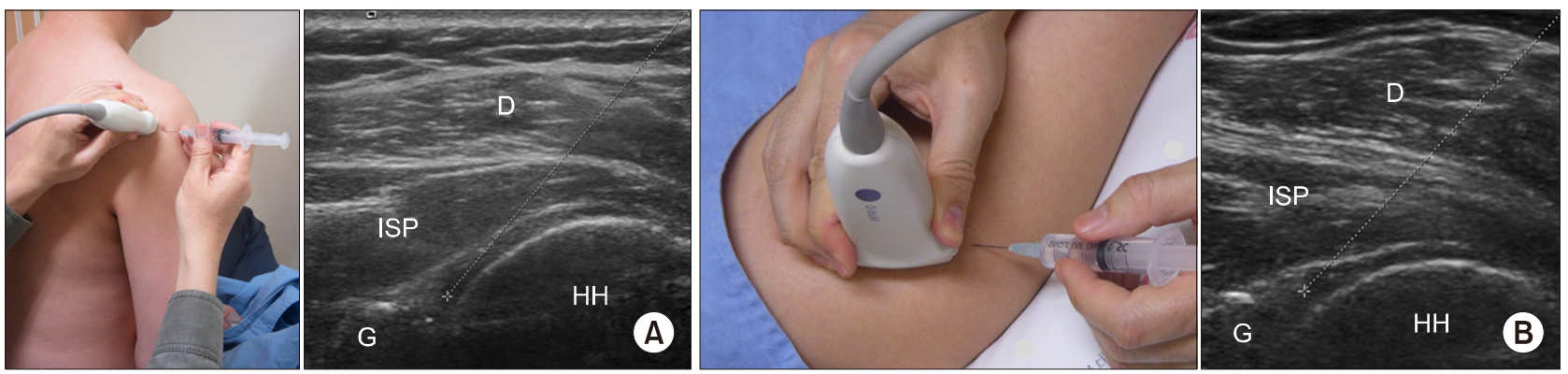

Figure 4. Ultrasonography guided glenohumeral joint injection using posterior approach in seated (A) and prone (B) position. D: deltoid, ISP: infraspinatus muscle, G: glenoid, $\mathrm{HH}$ : humeral head. 

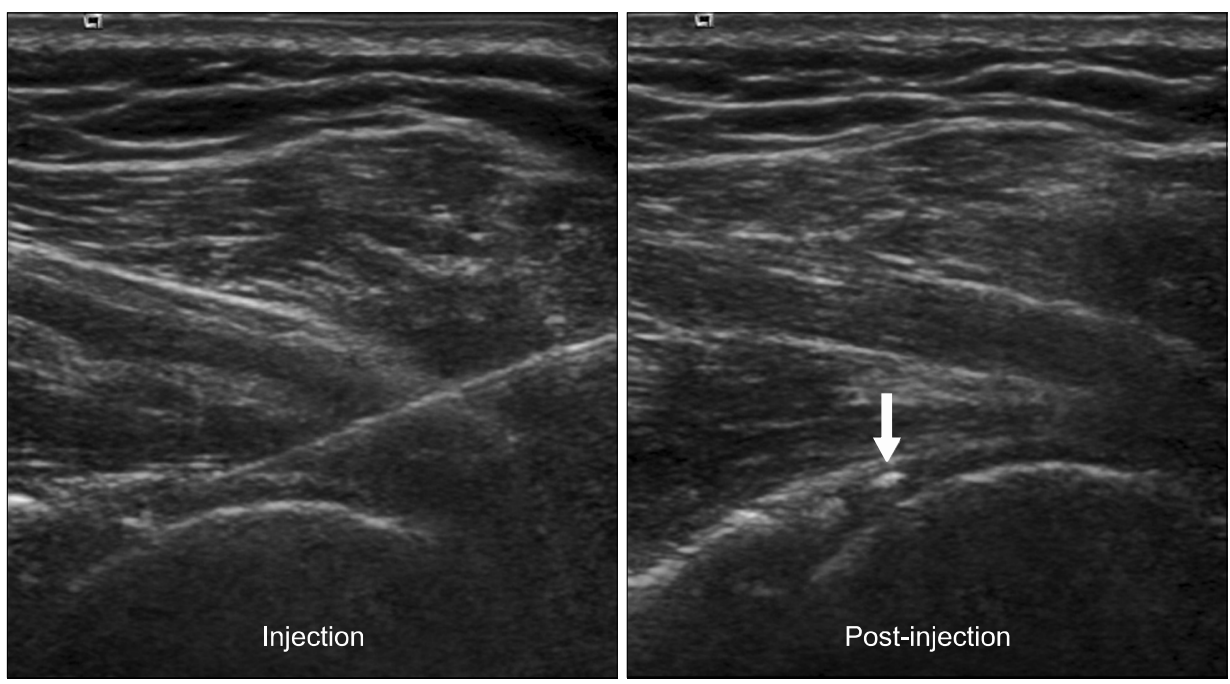

Figure 5. Ultrasonography guided glenohumeral joint injection using posterior approach in prone position. The needle tip is targeted at the point between the posterior labrum and posterior humeral head cartilage. The hyperechoic triamcinolone crystal (arrow) is detected in posterior glenohumeral joint after injection.

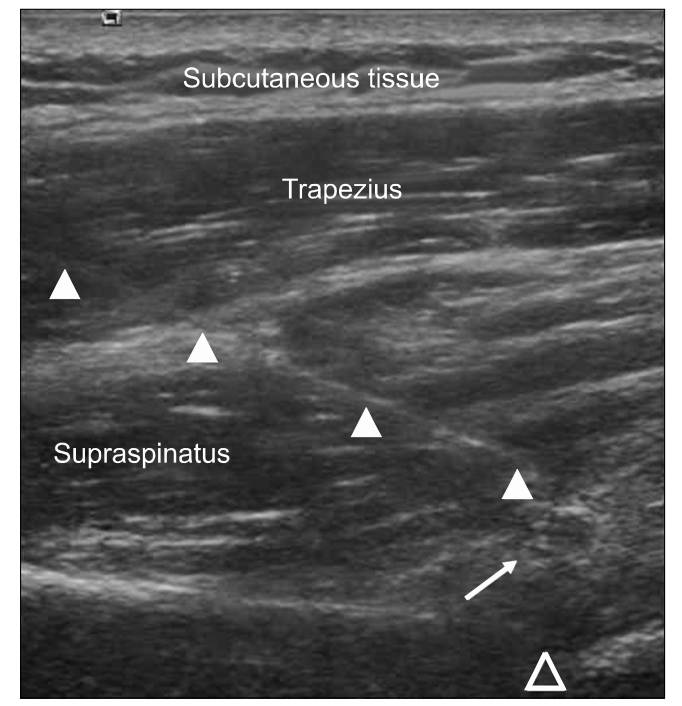

Figure 6. Distal (classic) suprascapular nerve block under ultrasonography guidance. Needle is (arrow heads) approaching on the suprascapular nerve and artery (arrow) in suprascapular notch (open arrow head).

humeral joint. A suprascapular nerve block using a local anesthetic effectively treats painful shoulder conditions such as FS, particularly by providing short-term pain relief to facilitate shoulder exercises [24]. Ultrasonographyguided suprascapular nerve block can be performed via a proximal or distal approach. The classic (distal) approach to block the suprascapular nerve targets the suprascapular nerve in the suprascapular notch through which the nerve enters the suprascapular fossa (Figure 6). In this location, ultrasonography visualization of the suprascapular nerve is difficult because it is located deeply within the suprascapular fossa. Ultrasonography-guided

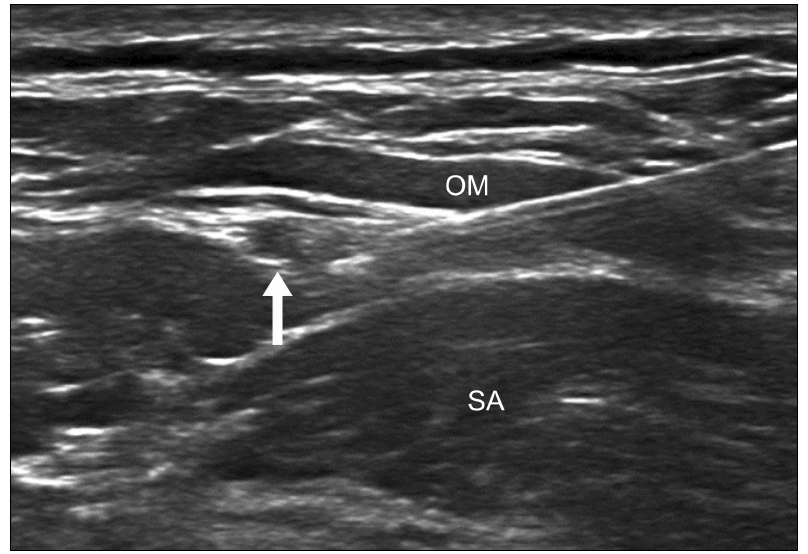

Figure 7. Proximal suprascapular nerve block under ultrasonography guidance, Needle tip (arrow) is located beneath proximal suprascapular nerve between omohyoid muscle $(\mathrm{OM})$ and serratus anterior muscle (SA).

suprascapular nerve block via the proximal approach scores over the distal approach because the needle tip and the suprascapular nerve are easily identified with the proximal approach owing to the superficial position of the suprascapular nerve in this location, it is associated with a higher level of accuracy, a small amount of local anesthetic is adequate to successfully complete the nerve block, and it provides more effective pain relief [25]. Ultrasonography of the anterior neck (transverse view across the anterior surface of the neck) shows the suprascapular nerve originating from the upper trunk of the brachial plexus, and its course can be traced as it passed beneath the inferior belly of the omohyoid muscle [26]. In-plane ultrasonography-guided selective proximal suprascapular nerve block is performed by injecting $2 \mathrm{~mL}$ of 

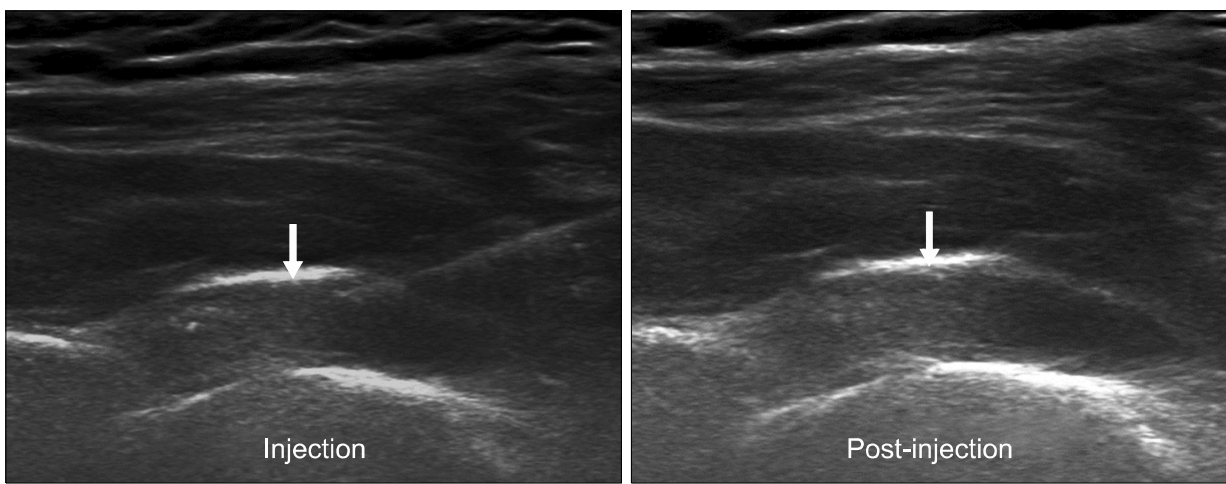

Figure 8. Glenohumeral distension arthrography under ultrasonography guidance. Capsular distension of posterior glenohumeral joint (arrow) is noted during and after distension arthrography. lidocaine $(10 \mathrm{mg} / \mathrm{mL})$ around the nerve lying under the omohyoid muscle (Figure 7).

\section{3) Ultrasonography-guided glenohumeral distention arthrography}

Glenohumeral distention arthrography is an effective treatment strategy in patients with a greater degree of limitation than pain (patients presenting in the freezing or frozen stage). This procedure is performed under fluoroscopic or ultrasonography guidance (Figure 8 ). The intracapsular pressure and volume of glenohumeral joint are gradually increased via the injection of a saline solution containing local anesthetics with/without corticosteroid and contrast material until capsular rupture occurs $[22,27,28]$.The combination of distention arthrography and manipulation under general anesthesia resulted in rapid pain relief and improve shoulder range of motion in 4 to 6 weeks after intervention [29]. The combination of distension arthrography and translational mobilization and Cyriax's capsular stretching was shown to significantly reduce shoulder pain and improve shoulder passive range of motion without serious complications in patients presenting in the freezing or frozen stage of FS [28]. Combination treatment may be therapeutically more effective than distention arthrography alone for the treatment of FS.

\section{4) Combination of multiple treatments}

The recent management protocol used in clinical practice to improve shoulder passive range of motion recommend the suprascapular nerve block followed by injection of an intra-articular corticosteroid and a local anesthetics with brisement normal saline volume dilation, and finally manipulation of the shoulder [30]. This protocol is effective in significant improvement in the shoulder passive range of motion immediately after the procedure. Our treatment protocol used in patients presenting with the freezing or frozen stage of FS consists of an ultrasonography-guided proximal suprascapular nerve block and subacromial subdeltoid bursa injection with local anesthetic, fluoroscopic- or ultrasonography-guided glenohumeral distention arthrography, and finally, modified translation mobilization with Cyriax's capsular stretching.

\section{CONCLUSION}

Ultrasonography, ultrasonography-guided glenohumeral joint injection, suprascapular nerve block, and distention glenohumeral arthrography can accurately diagnose and treat FS. Selecting a treatment strategy for FS needs careful attention to patient's symptoms and the stage of FS because patients require an individualized therapeutic strategy.

\section{CONFLICT OF INTEREST}

No potential conflict of interest relevant to this article was reported.

\section{REFERENCES}

1. Duplay S. [De la peri-arthrite scapulo-humerale et des raideurs de l'epaule qui en sont la consequence]. Arch Gen Med 1872;20:513-42. French.

2. Codman EA. The shoulder: rupture of the supraspinatus tendon and other lesions in or about the subcromial bursa. Boston, Thomas Todd, 1934, p. 216-24.

3. Neviaser JS. Adhesive capsulitis of the shoulder. A study of the pathological findings in periarthritis of the shoulder. J Bone Joint Surg Am 1945;27:211-22.

4. Lundberg BJ. The frozen shoulder. Clinical and radiographical observations. The effect of manipulation under general anesthesia. Structure and glycosaminoglycan content of the joint capsule. Local bone metabolism. Acta Orthop Scand Suppl 1969;119:1-59. 
5. Zuckerman JD, Rokito A. Frozen shoulder: a consensus definition. J Shoulder Elbow Surg 2011;20:322-5.

6. Reeves B. The natural history of the frozen shoulder syndrome. Scand J Rheumatol 1975;4:193-6.

7. Hannafin JA, Chiaia TA. Adhesive capsulitis: a treatment approach. Clin Orthop Relat Res 2000;(372):95-109.

8. Cyriax JH. Textbook of orthopaedic medicine. Vol. 1, diagnosis of soft tissue lesions. 8th ed. London, Baillière Tindall, 1982, p. 134-5.

9. Loyd JA, Loyd HM. Adhesive capsulitis of the shoulder: arthrographic diagnosis and treatment. South Med J 1983;76: 879-83.

10. Neviaser TJ. Arthrography of the shoulder. Orthop Clin North Am 1980;11:205-17.

11. Connell D, Padmanabhan R, Buchbinder R. Adhesive capsulitis: role of MR imaging in differential diagnosis. Eur Radiol 2002;12:2100-6

12. Mengiardi B, Pfirrmann CW, Gerber C, Hodler J, Zanetti M. Frozen shoulder: MR arthrographic findings. Radiology 2004;233:486-92.

13. Homsi C, Bordalo-Rodrigues M, da Silva JJ, Stump XM. Ultrasound in adhesive capsulitis of the shoulder: is assessment of the coracohumeral ligament a valuable diagnostic tool? Skeletal Radiol 2006;35:673-8.

14. Kwon DR, Kim MY, Chae YJ, Park JS, Kim JS, Yi TI. Comparison of coracohumeral ligament thickness between asymptomatic shoulders and adhesive capsulitis in Korean. J Korean Acad Rehabil Med 2009;33:392-5.

15. Lee JC, Sykes C, Saifuddin A, Connell D. Adhesive capsulitis: sonographic changes in the rotator cuff interval with arthroscopic correlation. Skeletal Radiol 2005;34:522-7.

16. McNally EG, Rees JL. Imaging in shoulder disorders. Skeletal Radiol 2007;36:1013-6.

17. Walmsley S, Osmotherly PG, Walker CJ, Rivett DA. Power doppler ultrasonography in the early diagnosis of primary/idiopathic adhesive capsulitis: an exploratory study. J Manipulative Physiol Ther 2013;36:428-35.

18. Park GY, Park JH, Kwon DR, Kwon DG, Park J. Do the findings of magnetic resonance imaging, arthrography, and ultrasonography reflect clinical impairment in patients with idiopathic adhesive capsulitis of the shoulder? Arch Phys Med Rehabil 2017;98:1995-2001.

19. Park GY, Kwon DR. Application of real-time sonoelastography in musculoskeletal diseases related to physical medi- cine and rehabilitation. Am J Phys Med Rehabil 2011;90: 875-86.

20. Wu CH, Chen WS, Wang TG. Elasticity of the coracohumeral ligament in patients with adhesive capsulitis of the shoulder. Radiology 2016;278:458-64.

21. Park GY, Lee JH, Kwon DG. Ultrasonographic measurement of the axillary recess thickness in an asymptomatic shoulder. Ultrasonography 2017;36:139-43.

22. Park GY, Hwang SE. Comparison of intraarticular steroid injection with and without capsular distension in adhesive capsulitis of the shoulder. J Korean Acad Rehabil Med 2000;24:1174-9.

23. OhJH, Oh CH, Choi JA, Kim SH, Kim JH, Yoon JP. Comparison of glenohumeral and subacromial steroid injection in primary frozen shoulder: a prospective, randomized short-term comparison study. J Shoulder Elbow Surg 2011;20:1034-40.

24. Dahan TH, Fortin L, Pelletier M, Petit M, Vadeboncoeur R, Suissa S. Double blind randomized clinical trial examining the efficacy of bupivacaine suprascapular nerve blocks in frozen shoulder. J Rheumatol 2000;27:1464-9.

25. Ko KP, Kang DH, Shin BK. The proximal approach in an ultrasound-guided suprascapular nerve block. J Korean Orthop Assoc 2017;52:521-8.

26. Rothe C, Steen-Hansen C, Lund J, Jenstrup MT, Lange KH. Ultrasound-guided block of the suprascapular nerve - a volunteer study of a new proximal approach. Acta Anaesthesiol Scand 2014;58:1228-32.

27. Buchbinder R, Green S, Forbes A, Hall S, Lawler G. Arthrographic joint distension with saline and steroid improves function and reduces pain in patients with painful stiff shoulder: results of a randomised, double blind, placebo controlled trial. Ann Rheum Dis 2004;63:302-9.

28. Park GY, Kwon DR, Kwon DG, Rim JH. Comparison of therapeutic effectiveness between shoulder distention arthrography with translation mobilization and distention arthrography alone in patients with frozen shoulder. Ann Rehabil Med 2018;42:76-84.

29. Ekelund AL, Rydell N. Combination treatment for adhesive capsulitis of the shoulder. Clin Orthop Relat Res 1992: 105-9.

30. Mitra R, Harris A, Umphrey C, Smuck M, Fredericson M. Adhesive capsulitis: a new management protocol to improve passive range of motion. PM R 2009;1:1064-8. 